\title{
Cost and Utility of Routine Contrast-Enhanced Neck MRA in a Pediatric MRI Stroke Evaluation Protocol
}

\author{
(D) A. Baltensperger, (DD. Mirsky, (D). Maloney, (DI. Neuberger, (D). Fenton, (D) T. Bernard, (D). Borgstede, and (D) N. Stence
}

\begin{abstract}
BACKGROUND AND PURPOSE: Cervical arterial dissection is one of the frequent causes of pediatric arterial ischemic stroke. Out of concern for missing cervical arterial dissection in patients in whom pediatric stroke is suspected, our tertiary children's hospital added contrast-enhanced 3D neck MR angiography to every pediatric stoke work-up. This research investigated whether the routine use of contrast-enhanced neck MRA in our MR imaging stroke protocol ever detected a cervical arterial abnormality when the DWI, SWI/gradient recalled-echo, or circle of Willis MRA findings from the brain MR imaging were reported as normal.
\end{abstract}

MATERIALS AND METHODS: The institutional PACS data base was searched for stroke protocol MRIs that included DWI, gradient recalled-echo or SWI, circle of Willis MRA, and 3D contrast-enhanced neck MRA in patients younger than 18 years of age with examinations performed between September 2010 and June 2017.

RESULTS: In only a single case (0.15\%) were the DWI, SWI/gradient recalled-echo, or circle of Willis MRA findings all separately reported as normal and the contrast-enhanced neck MRA findings reported as abnormal. To reach these findings, we screened 681 patients, which would have resulted in an estimated $>\$ 200,000$ in Medicare charges and $\$ 80,000$ in of out-of-pocket cost to patients.

CONCLUSIONS: In our large series, the addition of a routine contrast-enhanced neck MRA to our pediatric stroke MR imaging protocol was of extremely low yield. We believe the use of neck MRA should reasonably be limited to cases in which abnormalities are initially detected on standard brain sequences or to patients with atypical presentation or recurrent pathology.

ABBREVIATIONS: $\mathrm{AIS}=$ arterial ischemic stroke; $\mathrm{CAD}=$ cervical arterial dissection; $\mathrm{CE}=$ contrast-enhanced; CoW $=$ circle of Willis; GRE $=$ gradient recalled-echo; VEN_BOLD $=$ venous blood oxygen level-dependent

A cute ischemic stroke (AIS) accounts for about half of all strokes in children, ${ }^{1}$ and most survivors have life-long disability. ${ }^{2}$ Cervical arterial dissection (CAD) is a major cause of pediatric AIS, occurring in 2.5 children per 100,000 per year and accounting for $5 \%-25 \%$ of childhood-onset AIS. ${ }^{3}$ Furthermore,

Received June 17, 2019; accepted after revision September 18.

From the Department of Pediatrics (A.B., T.B.), Section of Child Neurology, Department of Radiology (D.M., J.M., I.N., L.F., N.S.), Section of Pediatric Radiology, and Department of Radiology (J.B.), University of Colorado School of Medicine, Aurora, Colorado.

T.B.'s work on this project was supported by the Health Resources and Services Administration of the US Department of Health and Human Services under No. 2H30MC24049, Mountain States Hemophilia Network.

This information or content and conclusions are those of the authors and should not be construed as the official position or policy of nor should any endorsements be inferred by Health Resources and Services Administration, US Department of Health and Human Services, or the US Government.

Please address correspondence to Nicholas Stence, MD, Department of Radiology, Children's Hospital Colorado, 13123 E 16th Ave, Aurora, CO 80045; e-mail: nicholas.stence@ucdenver.edu

- Indicates open access to non-subscribers at www.ajnr.org

http://dx.doi.org/10.3174/ajnr.A6315 children with AIS secondary to CAD have a significant recurrence risk, estimated at $19 \%-20 \%$, and a diagnosis of CAD has the potential to change management. ${ }^{4,5}$

Children with acute-onset focal neurologic deficits present a unique clinical challenge. AIS is the most common diagnosis for an adult presenting with an acute-onset focal neurologic deficit, occurring in $73 \%$ of cases. However, AIS accounts for only $7 \%$ of children presenting emergently with neurologic deficits, with migraines, seizures, and Bell palsy occurring more frequently. ${ }^{6}$ Therefore, while AIS is an important, treatable, and common etiology of this presentation in children, imaging protocols in this setting should also evaluate other etiologies.

In most pediatric stroke centers, MR imaging/MRA has become the first-line imaging technique for pediatric patients with suspected AIS because it is noninvasive and free of radiation, while effectively evaluating AIS, dissection, and other neurologic diseases. MR imaging protocols usually include diffusionweighted, FLAIR, and T1 images of the brain and T1 or T2 fatsaturated axial imaging through the neck. MRA typically includes 
3D time-of-flight MRA of the head and neck (from the aortic arch through the circle of Willis). ${ }^{7}$

Recent guidelines regarding the urgent management of stroke in children include recommendations for MR imaging with axial DWI, fluid-attenuated inversion recovery, susceptibility-weighted imaging, and time-of-flight MR angiography of the head and neck. ${ }^{2}$ More recently in 2017, the International Pediatric Stroke Study Neuroimaging Consortium and the Pediatric Stroke Neuroimaging Consortium published recommendations on imaging pathways for children presenting with stroke, endeavoring to further standardize the practice. The publication argues that because some arteriopathies in childhood affect the cervical vessels while others, the intracranial vasculature, vascular imaging of the head and neck ought to be performed in most cases, specifically including at the time of initial MR imaging, an MRA of the head and neck to evaluate arteriopathy, thrombus, or findings that require independent prompt treatment (ie, cervical artery dissection). ${ }^{8}$

However, equipoise remains on institutional practices for imaging patients with suspected stroke, with an unclear prevalence of abnormal neck imaging findings in the setting of normal brain imaging findings to bolster such practices. There is no comprehensive evaluation of the rates of abnormal neck vessel imaging findings in all-comers pediatric patients to stroke protocol imaging. The Vascular effects of Infection in Pediatric Stroke (VIPS) study identified only one-half of the 355 patients as having undergone cervical imaging at stroke ictus. ${ }^{9}$ More data are needed to drive an effective determination of the clinical utility of neckvessel imaging in pediatric stroke and further standardize our current practices. An appreciated low utility could realistically minimize a potentially unnecessary (as routine) diagnostic component of stroke protocol that would benefit resource use within the institution as well as expedition of appropriate patient care.

Out of concern for missing CAD in children when AIS is suspected, in 2010, our tertiary children's hospital (Children's Hospital Colorado, Aurora, Colorado) added contrast-enhanced neck MR angiography (CE-MRA) to every MR imaging ordered for a pediatric stroke work-up, with the option to limit the examination to DWI, gradient recalled-echo (GRE)/VEN_BOLD, and circle of Willis (CoW) MRA only if an acute stroke was seen and administration of tPA was considered. CE-MRA was chosen because it was much faster than $2 \mathrm{D}$ or $3 \mathrm{D}$ time-of-flight noncontrast MRA (30 seconds versus 5-10 minutes). Since the adoption of this MR imaging pediatric stroke protocol, the newly discovered issue of gadolinium deposition has caused us to re-evaluate our neuroimaging protocols that use gadolinium contrast agents. For this reason and to ensure best practices, we undertook a retrospective quality-improvement project to determine whether the routine use of a neck CE-MRA in our MR imaging stroke protocol was indeed necessary, specifically in the subset of children in whom the DWI, GRE/VEN_BOLD, or CoW MRA findings from the brain MR imaging were reported as normal.

\section{MATERIALS AND METHODS \\ Patients}

The study was a local institutional review board-approved retrospective assessment of MR imaging stroke protocol performed at our hospital between September 2010 and June 2017. The institutional PACS database at Children's Hospital Colorado was searched using Montage (Montage Healthcare Solutions, Philadelphia, Pennsylvania) for stroke protocol MRIs that included DWI, GRE, or VEN_BOLD; circle of Willis MRA; and neck CE-MRA in patients younger than 18 years of age.

We reviewed reports and collected the following data: accession number, patient age, examination date, any abnormal findings on examinations; abnormal findings on DWI (stroke or other); stroke location if applicable (anterior cerebral artery, MCA, posterior cerebral artery, cerebellum, brain stem); abnormal findings on CoW MRA (stenosis or occlusion or other), GRE or VEN_BOLD (bleeding or venous distension or other), neck MRA (stenosis or occlusion or other), or those identified on different pulse sequences; and eventual clinical diagnosis.

\section{CE-MRA Protocol}

The neck CE-MRA sequences were acquired on either a $1.5 \mathrm{~T}$ scanner (Avanto; Siemens, Erlangen, Germany) or a 3T scanner (Ingenia; Philips Healthcare, Best, the Netherlands). The 1.5T neck CE-MRA sequence used TR/TE, 3.17/1.1 ms; acquisition matrix, $110 \times 384 ; 72$ slices; $1.2-\mathrm{mm}$ section thickness with no gap. The $3 \mathrm{~T}$ neck CE-MRA sequence used TR/TE, 4/1.4 ms; acquisition matrix, $251 \times 300$; 70 slices; $1.8-\mathrm{mm}$ section thickness with $-0.9 \mathrm{~mm}$ gap. Neck CE-MRA was performed with bolus tracking after the administration of $0.1 \mathrm{mmol} / \mathrm{kg}$ body weight of gadolinium-based contrast agent (MultiHance, $0.5 \mathrm{mmol} / \mathrm{mL}$, gadobenate dimeglumine; Bracco Diagnostics, Princeton, New Jersey) at a rate of $2 \mathrm{~mL} / \mathrm{s}$.

\section{Cost-Effectiveness Analysis}

The Medicare Physician Fee Schedule global charge for a postcontrast cervical MRA (CPT 70548) is $\$ 311.80$ (for 2019), which underestimates private payer reimbursement and generally overestimates Medicaid reimbursement. Given that most commercial payer subscribers pay, on average, $20 \%$ coinsurance after the deductible and because a typical commercial contract can be up to twice Medicare rates, our estimates for out-of-pocket expenses for these families range from approximately $\$ 62$ to $\$ 125$.

\section{RESULTS}

The search result yielded 1147 examinations. Following exclusion of 10 examinations for excessive artifacts or incomplete documentation, there were 1137 examinations in 888 unique patients (522 males; average age, 10.8 years).

In only a single case (a 15-year-old girl with an unusual history of episodic cervical ICA vasospasm) were the DWI, SWI/ GRE, or CoW MRA findings all separately reported as normal and the neck CE-MRA findings reported as abnormal for a cervical arterial abnormality. With a total of 681 studies without any identified abnormalities on brain imaging, this case represented $0.15 \%$ of those total studies. In comparison, 456 cases reported abnormal findings on DWI, GRE/VEN_BOLD, or CoW MRA, with $130(28.5 \%)$ of those cases having abnormal neck vessel imaging findings (absolute proportion difference, 28.35; 95\% CI, 24.34-32.66; $P<.001)$. 
By means of our estimates of cost, the routine neck CE-MRA imaging performed in the 681 brain studies that were reported as having normal findings would have resulted in an estimated $\$ 212,335.80$ in Medicare payments and potentially from $\$ 42,222$ to $\$ 85,125$ in patient out-of-pocket costs.

\section{DISCUSSION}

In our large series of $>660$ children with strokelike symptoms, the addition of a routine neck CE-MRA to our pediatric stroke MR imaging protocol was of extremely low yield when DWI, GRE/VEN_BOLD, and MRA CoW findings were all normal, revealing only a single case of carotid pathology (0.15\%). Our number of 681 needed to screen patients, using a conservative Medicare cost estimate, would result in $>\$ 200,000$ of charges and $\$ 80,000$ of out of pocket cost to patients.

Additionally, when our protocol was initially devised in 2010, our use of neck CE-MRA in these patients was predicated on its relatively fast acquisition time of $\sim 30$ seconds. However, given the recently discovered and ongoing concerns over findings of gadolinium deposition in children, ${ }^{10}$ many pediatric centers are not administering contrast whenever possible. Substituting a noncontrast time-of-flight neck MRA could add 5-10 minutes of imaging time to an examination in which speed of imaging is often critical.

Since conducting this study, we have significantly limited the routine use of neck CE-MRA in our stroke imaging work-ups. However, in certain clinical circumstances, we believe it is still important to evaluate the cervical vessels, even when brain imaging findings are normal, particularly when symptoms may point to pathology in the posterior circulation, given the increased prevalence of vertebral dissection in these patients. ${ }^{4,7}$

\section{CONCLUSIONS}

On the basis of the extremely low yield of routine neck CE-MRA in a pediatric stroke MR imaging protocol when DWI, GRE/ VENOUS_BOLD, and CoW MRA findings are normal, we believe the use of a neck CE-MRA in the evaluation of pediatric stroke should reasonably be limited to cases in which abnormalities are initially detected on routine brain sequences or in patients with atypical presentations or recurrent pathology.

Disclosures: Austin Baltensperger-UNRELATED: Employment: United States Air Force. Laura Fenton-UNRELATED: Board Membership: Society for Pediatric Radiology, Board of Directors, Comments: No money was paid to me or the institution.

\section{REFERENCES}

1. Lynch JK, Hirtz DG, DeVeber G, et al. Report of the National Institute of Neurological Disorders and Stroke workshop on perinatal and childhood stroke. Pediatrics 2002;109:116-23 CrossRef Medline

2. Rivkin MJ, Bernard TJ, Dowling MM, et al. Guidelines for urgent management of stroke in children. Pediatr Neurol 2016;56:8-17 CrossRef Medline

3. Mackay MT, Wiznitzer M, Benedict SL, et al. Arterial ischemic stroke risk factors: the International Pediatric Stroke Study. Ann Neurol 2011;69:130-40 CrossRef Medline

4. Uohara MY, Beslow LA, Billinghurst L, et al. Incidence of recurrence in posterior circulation childhood arterial ischemic stroke. JAMA Neurol 2017;74:316-23 CrossRef Medline

5. Fullerton HJ, Wintermark M, Hills NK, et al. Risk of recurrent arterial ischemic stroke in childhood: a prospective international study. Stroke 2016;47:53-59 CrossRef Medline

6. Mackay MT, Chua ZK, Lee M, et al. Stroke and nonstroke brain attacks in children. Neurology 2014;82:1434-40 CrossRef Medline

7. Stence NV, Fenton LZ, Goldenberg NA, et al. Craniocervical arterial dissection in children: diagnosis and treatment. Curr Treat Options Neurol 2011;13:636 CrossRef Medline

8. Mirsky DM, Beslow LA, Amlie-Lefond C, et al. Pathways for neuroimaging of childhood stroke. Pediatr Neurol 2017;69:11-23 CrossRef Medline

9. Wintermark M, Hills NK, DeVeber GA, et al. Clinical and imaging characteristics of arteriopathy subtypes in children with arterial ischemic stroke: results of the VIPS study. AJNR Am J Neuroradiol 2017;38:2172-79 CrossRef Medline

10. Flood TF, Stence NV, Maloney JA, et al. Pediatric brain: repeated exposure to linear gadolinium-based contrast material is associated with increased signal intensity at unenhanced T1-weighted MR imaging. Radiology 2017;282:222-28 CrossRef Medline 